\title{
Comparison of the Effect of Parameterized Eddy Fluxes of Thickness and Potential Vorticity
}

\author{
V. O. IVCHENKO, S. DANILOV, AND J. SCHRÖTER \\ Alfred-Wegener-Institute for Polar and Marine Research, Bremerhaven, Germany
}

(Manuscript received 4 December 2013, in final form 19 May 2014)

\begin{abstract}
Parameterization of mesoscale eddies is an important problem of modern ocean dynamics and modeling. The most widely used scheme is the so-called Gent-McWilliams parameterization, which describes the eddyinduced transport of tracers, including temperature, density, and isopycnal thickness (TH). An alternative scheme, proposed by Green and Welander, deals with parameterizing eddy fluxes of potential vorticity (PV). Many recent studies propose using it, for it includes the effect of eddy Reynolds stresses that may influence mean flows. These two schemes are compared in the simplest configuration of two-layer quasigeostrophic channel flow, which enables analytical solutions for zonal-mean fields. It is shown how the parameterizations shape the zonally averaged zonal velocity profiles, with special attention paid to the role of the Reynolds stresses and momentum conservation. The zonally averaged zonal velocity profiles are sensitive to the amplitude and profiles of TH and PV diffusivities. For small enough diffusivities the TH parameterization may lead to solutions resembling those for the PV parameterization if it uses the diffusivity of the latter; that is, it may mimic the impact of the Reynolds stresses on the mean flow.
\end{abstract}

\section{Introduction}

There is a local maximum of kinetic energy on ocean mesoscales (Kamenkovich et al. 1986), which demands from numerical models either to resolve mesoscale features or parameterize them. Although the progress in computers makes global eddy-resolving simulations rather common, they still remain expensive for long enough integrations. In addition to this practical aspect, studies of eddy parameterization contribute to better understanding of ocean dynamics, remaining thereby in the focus of the modern physical oceanography.

Parameterization of eddy fluxes involves both the momentum fluxes (Reynolds stresses) and tracers fluxes (temperature, salinity, density, and other tracers). An important approach in the latter case is the popular scheme by Gent and McWilliams (1990) for eddy-induced thickness (TH) fluxes. This parameterization leads to substantial improvement in a number of oceanic features simulated with coarse models, especially in the Southern Ocean, where it ensures a deep ocean density distribution

Corresponding author address: V. O. Ivchenko, Alfred Wegener Institute for Polar and Marine Research, Bussestrasse 24, D-27570, Bremerhaven, Germany.

E-mail: vladimir.ivchenko@awi.de that is closer to the observed values, a sharper thermocline, eddy-induced cancellation of the Deacon cell, and a better representation of the penetration depth of chemical tracers originating in the atmosphere (see, e.g., Danabasoglu et al. 1994; Hirst and McDougall 1996). This scheme allows one to introduce the so-called bolus velocity and reformulate the basic equations with its assistance (see, e.g., Griffies 2004). The most popular eddy flux parameterization is that of downgradient transport (diffusive parameterization), when the eddy flux of a scalar variable is taken to be proportional to the negative of the gradient of the mean field. However, such a scheme can only be applied to conserved quantities. Momentum is a nonconserved quantity because of the presence of the pressure gradient; parameterizing such quantities presents a challenge. A satisfactory momentum parameterization is absent at present. In most cases, eddy fluxes of momentum are modeled as eddy viscosity (harmonic or biharmonic) that is not uncontroversial, as momentum fluxes can be countergradient and "sharpen" jet flows instead of diffusing them. The popularity of harmonic and biharmonic viscosities lies rather in their contribution to numerical stability than their realism (Harrison 1978; Killworth 1997).

Zonal jets are a common feature of geophysical flows (see, e.g., Rhines 1975; Danilov and Gurarie 2002). They are encountered in many places across the World Ocean. 
In the midlatitudes the multiple jets have rms zonal velocities estimated as about $6.9 \mathrm{~cm} \mathrm{~s}^{-1}$ and a meridional wavelength of about $300 \mathrm{~km}$ (Maximenko et al. 2005). Mesoscale eddies play the major role in creating and supporting such jets through the convergence of lateral eddy momentum fluxes (Dritschel and McIntyre 2008; Eden 2010).

Importantly, the parameterization of potential vorticity (PV) fluxes incorporates the Reynolds stresses, since they are included in the eddy PV fluxes together with fluxes of density [or, with high accuracy, TH]. Note also that there is a better physical base for diffusive parameterization of $\mathrm{PV}$, which is a conserved variable. This explains a growing interest in parameterizations of eddy PV fluxes (Treguier et al. 1997; Wardle and Marshall 2000; Eden and Greatbatch 2008; Eden 2010; Marshall and Adcroft 2010; Ringler and Gent 2011).

The main aim of this study is comparing/contrasting the PV and TH parameterizations in their effect on velocities, energy, and other variables and analyzing their dependence on respective diffusivities. The difference between solutions with $\mathrm{TH}$ and PV flux parameterizations comes mainly from the effect of Reynolds stresses (which are not considered, i.e., virtually neglected in the $\mathrm{TH}$ scheme) and constraints stemming from the momentum conservation [the parameterized PV fluxes must be compatible with the momentum balance, as stated by the Bretherton theorem (Bretherton 1966), and ensure a positive kinetic energy source in the eddy kinetic energy balance]. Ideally, such a comparison should be done for general 3D primitive equation dynamics, which still presents a challenge. The first step can be made by using strongly simplified quasigeostrophic dynamics in simple channel geometry, which can be treated analytically.

For the quasigeostrophic channel model, the integral constraints have been repeatedly discussed for both momentum (Bretherton 1966; McWilliams et al. 1978; Marshall 1981; Ivchenko 1987; Ivchenko et al. 2008, 2013, 2014) and energy (Ivchenko et al. 2013, 2014). Wood and McIntyre (2010) proved a theorem linking PV mixing and the negative change in angular momentum.

It is hoped that the analysis of analytical solutions here can provide some hints toward understanding more complex dynamics. A caveat of this study is that it cannot be directly applied to channels with varying topography. It is well known that zonal transport in a flat bottom channel can be an order of magnitude higher than in channels with variable topography. The selection of the coefficients for the parameterized eddy fluxes must be done carefully to avoid unphysical and artificially high zonal transport.

However, the use of the simplest possible geometry of flat bottom channel already allows for analytical solutions and better understanding of the eddy flow dynamics.

This paper is organized as follows. We first write down the basic equations for quasigeostrophic dynamics and introduce the TH and PV parameterizations (sections 24). In section 5, the difference between solutions is studied for both constant and meridionally varying diffusivities. Section 6 discusses solutions for velocities and parameterizations for coefficients based on the timeand zonal-mean equation for eddy quasigeostrophic enstrophy. Section 7 analyzes the Reynolds stresses diagnosed by comparing solutions for PV and $\mathrm{TH}$ parameterizations, and section 8 offers discussion and conclusions.

\section{Basic quasigeostrophic equation for the zonal channel}

\section{Equations}

We use the quasigeostrophic equations for the twolayer model (Holland 1978; McWilliams et al. 1978; Wolff et al. 1991):

$$
\frac{\partial \nabla^{2} \Psi_{1}}{\partial t}=J\left(f+\nabla^{2} \Psi_{1}, \Psi_{1}\right)-\frac{f_{0}}{H_{1}} w_{3 / 2}+1 / H_{1} \operatorname{curl}_{z} \tau+F_{1},
$$

$\frac{\partial \nabla^{2} \Psi_{2}}{\partial t}=J\left(f+\nabla^{2} \Psi_{2}, \Psi_{2}\right)+\frac{f_{0}}{H_{2}} w_{3 / 2}-\epsilon \operatorname{curl}_{z} \mathbf{v}_{2}+F_{2}$,

and

$$
\frac{\partial h_{3 / 2}}{\partial t}=J\left(h_{3 / 2}, \Psi_{3 / 2}\right)+w_{3 / 2},
$$

where $\Psi_{i}$ is the quasigeostrophic streamfunction, subscripts label the layers of constant mean thicknesses $H_{i}$, and $w_{3 / 2}$ is the vertical velocity of the interface. The term $F_{i}$ is the lateral friction that is further omitted in the analytical solution; the Jacobian operator is

$$
J(A, B)=-\frac{\partial A}{\partial y} \frac{\partial B}{\partial x}+\frac{\partial A}{\partial x} \frac{\partial B}{\partial y}
$$

$\epsilon$ is the linear bottom drag; and $\tau=\left(\tau_{x}, \tau_{y}\right)$ is the wind stress with the components $\tau_{x}=\tau_{0} \sin (\pi y / L)$ and $\tau_{y}=0$, where $\tau_{0}$ is the amplitude of wind stress, and $L$ is the width of the channel. The components of fluid velocity $\mathbf{v}_{i}=\left(u_{i}, v_{i}\right)$ are expressed as $u_{i}=-\partial \Psi_{i} / \partial y$ and $v_{i}=\partial \Psi_{i} / \partial x$, and $f$ and $f_{0}$ denote the Coriolis parameter and its reference value, respectively. The interfacial displacement $h_{3 / 2}$ is linked to streamfunction as 


$$
h_{3 / 2}=\frac{f_{0}}{g^{\prime}}\left(\Psi_{2}-\Psi_{1}\right) \text {, }
$$

where $g^{\prime}=g\left(\rho_{2}-\rho_{1}\right) / \rho_{0}$ is the reduced gravity, $g$ is the acceleration due to gravity, $\rho_{i}$ and $\rho_{0}$ are the density of layer $i$ and the reference density, respectively, and $\Psi_{3 / 2}$ is the streamfunction of the interface:

$$
\Psi_{3 / 2}=\frac{H_{1} \Psi_{2}+H_{2} \Psi_{1}}{H_{1}+H_{2}}
$$

Combining (1)-(3) results in two equations for quasigeostrophic potential vorticity (QPV) $q_{1}$ and $q_{2}$ :

$$
\begin{aligned}
& \frac{\partial q_{1}}{\partial t}+J\left(\Psi_{1}, q_{1}\right)=1 / H_{1} \operatorname{curl}_{z} \tau+F_{1}, \quad \text { and } \\
& \frac{\partial q_{2}}{\partial t}+J\left(\Psi_{2}, q_{2}\right)=-\epsilon \operatorname{curl}_{z} \mathbf{v}_{2}+F_{2} .
\end{aligned}
$$

The layerwise quasigeostrophic potential vorticities $q_{i}$ are given by

$$
\begin{aligned}
& q_{1}=\nabla^{2} \Psi_{1}+f-\frac{f_{0}^{2}}{g^{\prime} H_{1}}\left(\Psi_{1}-\Psi_{2}\right), \quad \text { and } \\
& q_{2}=\nabla^{2} \Psi_{2}+f+\frac{f_{0}^{2}}{g^{\prime} H_{2}}\left(\Psi_{1}-\Psi_{2}\right) .
\end{aligned}
$$

\section{Parameterization of eddy thickness fluxes (TH parameterization)}

Zonal and time averaging of (1)-(3) leads to the following three equations:

$$
\frac{\overline{\partial \nabla^{2} \Psi_{1}}}{\partial t}=0=\overline{J\left(f+\nabla^{2} \Psi_{1}, \Psi_{1}\right)}-\frac{f_{0}}{H_{1}} \overline{w_{3 / 2}}-1 / H_{1} \frac{\partial \overline{\tau_{x}}}{\partial y},
$$

$\overline{\frac{\partial \nabla^{2} \Psi_{2}}{\partial t}}=0=\overline{J\left(f+\nabla^{2} \Psi_{2}, \Psi_{2}\right)}+\frac{f_{0}}{H_{2}} \overline{w_{3 / 2}}+\epsilon \frac{\partial \overline{u_{2}}}{\partial y}, \quad$ and

$$
\frac{\partial \bar{h}_{3 / 2}}{\partial t}=0=\overline{J\left(h_{3 / 2}, \Psi_{3 / 2}\right)}+\overline{w_{3 / 2}} .
$$

The overbar here denotes the time and zonal average.

The Jacobian operator in (10)-(11) represents advection of the absolute vorticity (the sum of planetary and relative vorticities) by the mean flow and eddies; it can be rewritten as

$$
\begin{aligned}
& \overline{J\left(f+\nabla^{2} \Psi_{i}, \Psi_{i}\right)}=-\overline{\frac{\partial f}{\partial y} v_{i}}-\overline{u_{i} \frac{\partial \nabla^{2} \Psi_{i}}{\partial x}}-\overline{v_{i} \frac{\partial \nabla^{2} \Psi_{i}}{\partial y}}, \\
& i=1,2 .
\end{aligned}
$$

The mean meridional velocity drops to zero because of zonal averaging:

$$
\overline{v_{i}}=\frac{\partial \overline{\Psi_{i}}}{\partial x}=0 .
$$

The advection of the relative vorticity can be presented as

$$
\begin{aligned}
-\overline{u_{i} \frac{\partial \nabla^{2} \Psi_{i}}{\partial x}}-\overline{v_{i} \frac{\partial \nabla^{2} \Psi_{i}}{\partial y}=} & -\bar{u}_{i} \frac{\partial \nabla^{2} \overline{\Psi_{i}}}{\partial x}-\overline{v_{i}} \frac{\partial \nabla^{2} \overline{\Psi_{i}}}{\partial y} \\
& -\overline{u_{i}^{\prime} \frac{\partial \nabla^{2} \Psi_{i}^{\prime}}{\partial x}}-\overline{v_{i}^{\prime} \frac{\partial \nabla^{2} \Psi_{i}^{\prime}}{\partial y}}
\end{aligned}
$$

where the prime implies the eddy component, that is, the deviation from the time and zonal mean. However,

$$
\frac{\partial \nabla^{2} \overline{\Psi_{i}}}{\partial x}=0
$$

and the rhs of (15) becomes

$$
-\overline{-u_{i}^{\prime} \frac{\partial \nabla^{2} \Psi_{i}^{\prime}}{\partial x}}-\overline{v_{i}^{\prime} \frac{\partial \nabla^{2} \Psi_{i}^{\prime}}{\partial y}}=\overline{\nabla^{2} \Psi_{i}^{\prime}\left(\frac{\partial u_{i}^{\prime}}{\partial x}+\frac{\partial v_{i}^{\prime}}{\partial y}\right)}-\overline{\frac{\partial v_{i}^{\prime} \nabla^{2} \Psi_{i}^{\prime}}{\partial y}}
$$

The first term on the rhs of (17) is zero, since the horizontal divergence of horizontal velocity is zero in the quasigeostrophic approach. The last term on the rhs of (17) can be transformed as

$$
\begin{aligned}
-\overline{\frac{\partial v_{i}^{\prime} \nabla^{2} \Psi_{i}^{\prime}}{\partial y}} & =-\frac{\partial}{\partial y}\left(\overline{v_{i}^{\prime} \frac{\partial v_{i}^{\prime}}{\partial x}}-\overline{v_{i}^{\prime} \frac{\partial u_{i}^{\prime}}{\partial y}}\right)=-\frac{\partial}{\partial y}\left(\frac{1}{2} \frac{\overline{\partial v_{i}^{\prime 2}}}{\partial x}-\overline{v_{i}^{\prime} \frac{\partial u_{i}^{\prime}}{\partial y}}\right) \\
& =\frac{\partial^{2} \overline{v_{i}^{\prime} u_{i}^{\prime}}}{\partial y^{2}}-\frac{\partial}{\partial y} \overline{u_{i}^{\prime} \frac{\partial v_{i}^{\prime}}{\partial y}},
\end{aligned}
$$

by virtue of

$$
\frac{\partial \overline{v_{i}^{\prime 2}}}{\partial x}=0 .
$$

The last term in (18) is zero because

$$
-\frac{\partial}{\partial y} \overline{u_{i}^{\prime} \frac{\partial v_{i}^{\prime}}{\partial y}}=\frac{\partial}{\partial y} \overline{u_{i}^{\prime} \frac{\partial u_{i}^{\prime}}{\partial x}}=\frac{1}{2} \frac{\partial}{\partial y}\left(\frac{\partial \overline{u_{i}^{\prime 2}}}{\partial x}\right)=0 .
$$


As a result, (10)-(11) can be rewritten as

$$
\begin{aligned}
-\frac{\partial^{2} \overline{v_{1}^{\prime} u_{1}^{\prime}}}{\partial y^{2}}+\frac{f_{0}}{H_{1}} \overline{w_{3 / 2}}+1 / H_{1} \frac{\partial \overline{\tau_{x}}}{\partial y}=0, \\
\frac{\partial^{2} \overline{v_{2}^{\prime} u_{2}^{\prime}}}{\partial y^{2}}+\frac{f_{0}}{H_{2}} \overline{w_{3 / 2}}+\epsilon \frac{\partial{\overline{u_{2}}}_{\partial y}^{\mathrm{TH}}}{=}=0 .
\end{aligned}
$$

Beginning from these equations we use superscripts $\mathrm{TH}$ and PV to label solutions for zonally averaged zonal velocity $\bar{u}_{i}$ obtained with respective parameterizations. No superscript is used in expressions that are independent of the parameterization choice. By applying diffusive parameterization to the meridional thickness flux in (12),

$$
\overline{J\left(h_{3 / 2}, \Psi_{3 / 2}\right)}=-\frac{\partial \overline{v_{3 / 2}^{\prime} h_{3 / 2}^{\prime}}}{\partial y}=\frac{\partial}{\partial y}\left(K_{H} \frac{\partial \bar{h}_{3 / 2}}{\partial y}\right),
$$

we rewrite (12) as

$$
\frac{\partial}{\partial y}\left(K_{H} \frac{\partial \bar{h}_{3 / 2}}{\partial y}\right)=-\bar{w}_{3 / 2},
$$

where $K_{H}$ is the coefficient of eddy thickness diffusivity (CT).

Using (21), (22), and (24), after standard manipulations, one can derive the energy balance equations for the mean and eddy mechanical (i.e., sum of kinetic and available potential) energies. Both balances will contain the term $K_{H}\left(\partial \bar{h}_{3 / 2} / \partial y\right)^{2}$, but with the opposite signs. This term represents the parameterized effect of baroclinic instability, that is, the mechanism transferring a part of mean available energy to eddies. This transfer should be positive (from mean to eddies) to maintain the eddies, which is the case under a trivial constraint that $K_{H}$ is positive.

The mean mechanical energy is generated by wind, dissipated by the bottom friction, and transferred to the eddy part of the mechanical energy.

Combining (21) and (22) and integrating the result in the meridional direction, we get the expression for the zonally averaged zonal velocity in the lower layer:

$$
\bar{u}_{2}^{\mathrm{TH}}=\frac{1}{\epsilon H_{2}} \tau_{x}-\frac{H_{1}}{\epsilon H_{2}} \frac{\partial \overline{v_{1}^{\prime} u_{1}^{\prime}}}{\partial y}-\frac{1}{\epsilon} \frac{\partial \overline{v_{2}^{\prime} u_{2}^{\prime}}}{\partial y} .
$$

The constant of integration is set to zero because the mean zonal velocities are zero on boundaries owing to the sinusoidal wind stress selected here (Marshall 1981; Ivchenko et al. 1997).

A combination of (24) with (21) followed by integration in the meridional direction yields

$$
-\frac{\partial \overline{v_{1}^{\prime} u_{1}^{\prime}}}{\partial y}+\frac{1}{H_{1}} \tau_{x}=\frac{f_{0}}{H_{1}} K_{H} \frac{\partial \bar{h}_{3 / 2}}{\partial y} .
$$

Because

$$
\frac{\partial \bar{h}_{3 / 2}}{\partial y}=\frac{f_{0}}{g^{\prime}}\left(\bar{u}_{1}^{\mathrm{TH}}-\bar{u}_{2}^{\mathrm{TH}}\right),
$$

the vertical shear of the mean zonal velocity is

$$
\left(\bar{u}_{1}^{\mathrm{TH}}-\bar{u}_{2}^{\mathrm{TH}}\right)=\frac{g^{\prime}}{f_{0}^{2} K_{H}} \tau_{x}-\frac{H_{1} g^{\prime}}{f_{0}^{2} K_{H}} \frac{\partial \overline{v_{1}^{\prime} u_{1}^{\prime}}}{\partial y} .
$$

The last expression together with (25) gives the expression for the zonally averaged zonal velocity in the upper layer:

$$
\begin{aligned}
\bar{u}_{1}^{\mathrm{TH}}= & \left(\frac{1}{\epsilon H_{2}}+\frac{g^{\prime}}{f_{0}^{2} K_{H}}\right) \tau_{x}-\left(\frac{H_{1}}{\epsilon H_{2}}+\frac{H_{1} g^{\prime}}{f_{0}^{2} K_{H}}\right) \frac{\partial \overline{v_{1}^{\prime} u_{1}^{\prime}}}{\partial y} \\
& -\frac{1}{\epsilon} \frac{\partial \overline{v_{2}^{\prime} u_{2}^{\prime}}}{\partial y} .
\end{aligned}
$$

Solutions (25) and (29) depend on the divergence of Reynolds stresses that can, as follows from eddy-resolving numerical simulations, substantially reshape the zonally averaged zonal velocity profiles (McWilliams et al. 1978; Wolff et al. 1991; Olbers 2005). However, there are no clear physical principles for parameterizing the Reynolds stresses on their own. As mentioned above, lateral viscous mixing used in numerical simulation rather smooths grid-scale features than provides a parameterization. The contribution of lateral viscosity can as a rule be neglected as compared to the impact of the bottom drag (Ivchenko et al. 1996; Stevens and Ivchenko 1997). We will therefore disregard the Reynolds stresses in the first part of this study. The diffusivity $K_{H}$ in expression (29) can take any value. We will use both a constant value of $K_{H}$ for comparing solutions with those found in the framework of PV parameterization and prescribed meridional profiles that resemble the behavior seen in eddyresolving experiments (see, e.g., Fig. 12 in McWilliams and Chow 1981):

$$
K_{H}=A_{H} F_{\mathrm{TH}}(y),
$$

where $A_{H}=$ const is the amplitude and $F_{\mathrm{TH}}$ is the normalized profile, $1 / L \int_{0}^{L} F_{\mathrm{TH}} d y=1$ (see Fig. 1).

\section{Parameterization of eddy potential vorticity fluxes}

\section{a. General expressions}

Taking the zonal-time average of (6)-(9) results in 


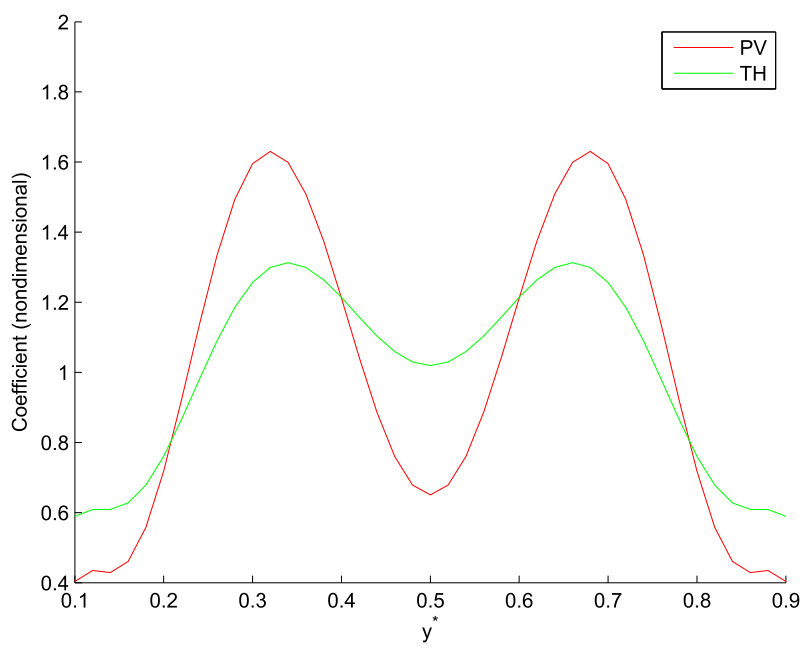

FIG. 1. Normalized coefficients for PV and TH parameterization. The meridional domain length is normalized to 1 .

$$
\begin{aligned}
& \frac{\partial \bar{q}_{1}}{\partial t}=0=-\frac{\partial}{\partial y} \overline{v_{1}^{\prime} q_{1}^{\prime}}-1 / H_{1} \frac{\partial}{\partial y} \bar{\tau}_{x}+\bar{F}_{1}, \\
& \frac{\partial \bar{q}_{2}}{\partial t}=0=-\frac{\partial}{\partial y} \overline{v_{2}^{\prime} q_{2}^{\prime}}+\epsilon \frac{\partial}{\partial y} \overline{u_{2}}+\overline{F_{2}}, \\
& \bar{q}_{1}=-\frac{\partial}{\partial y} \bar{u}_{1}+f-\frac{f_{0}^{2}}{g^{\prime} H_{1}}\left(\bar{\Psi}_{1}-\bar{\Psi}_{2}\right), \quad \text { and } \\
& \bar{q}_{2}=-\frac{\partial}{\partial y} \bar{u}_{2}+f+\frac{f_{0}^{2}}{g^{\prime} H_{2}}\left(\bar{\Psi}_{1}-\bar{\Psi}_{2}\right) .
\end{aligned}
$$

To proceed further analytically, we assume a diffusive parameterization for eddy fluxes of QPV in each layer (see Green 1970; Welander 1973):

$$
\overline{v_{i}^{\prime} q_{i}^{\prime}}=-K_{i} \frac{\partial \bar{q}_{i}}{\partial y}, \quad i=1,2,
$$

where $K_{i} \geq 0$ is the respective coefficient of QPV diffusivity abbreviated further as CPV. These parameterizations, when substituted into (31) and (32), lead to a closed system that can be solved for any $K_{i}$. However, the solutions should satisfy certain integral constraints, which in turn restrict the admissible values for $K_{i}$.

In a flat bottom case, the meridional integral of the depth-integrated meridional QPV flux is zero, which implies momentum conservation and makes the statement of the Bretherton theorem (Bretherton 1966; McWilliams et al. 1978; Marshall 1981; Ivchenko et al. 1997):

$$
\int_{0}^{L}\left(H_{1} \overline{v_{1}^{\prime} q_{1}^{\prime}}+H_{2} \overline{v_{2}^{\prime} q_{2}^{\prime}}\right) d y=0 .
$$

From (31), (32), and (36), it follows that the wind stress forcing is balanced by the bottom drag:

$$
\int_{0}^{L}\left(\tau_{x}-H_{2} \epsilon \overline{u_{2}}\right) d y=0 .
$$

Note that the expression (37) does not rely on eddy parameterizations and is an integral conservation law. It could be derived from (25) by meridional integration to eliminate the contribution from the Reynolds stresses.

Substitution of (35) in (36) leads to

$$
\int_{0}^{L}\left(K_{1} H_{1} \frac{\partial \overline{q_{1}}}{\partial y}+K_{2} H_{2} \frac{\partial \overline{q_{2}}}{\partial y}\right) d y=0 .
$$

This expression relates to the so-called first instability criterion of Pedlosky: "the potential vorticity gradient must somewhere change its sign for instability to occur" (Pedlosky 1964, ; Ivchenko et al. 2014).

Expression for the total eddy mechanical energy based on the parameterization of eddy PV fluxes provides insight into the exchange between the mean and eddy parts of mechanical energy.

To derive it, we multiply the basic QPV equations [(6) and (7)] by $H_{1} \Psi_{1}^{\prime}$ and $H_{2} \Psi_{2}^{\prime}$, respectively, and sum them to obtain

$$
\begin{aligned}
& \frac{\partial}{\partial t}\left(K_{1}^{\mathrm{ed}}+K_{2}^{\mathrm{ed}}+P^{\mathrm{ed}}\right)+H_{1} \frac{\partial}{\partial y}\left(\overline{\Psi_{1}^{\prime} \frac{\partial u_{1}^{\prime}}{\partial t}}\right)+H_{2} \frac{\partial}{\partial y}\left(\overline{\Psi_{2}^{\prime} \frac{\partial u_{2}^{\prime}}{\partial t}}\right)+\left(H_{1} \bar{u}_{1} \overline{v_{1}^{\prime} q_{1}^{\prime}}+H_{2} \bar{u}_{2} \overline{v_{2}^{\prime} q_{2}^{\prime}}\right) \\
& \quad-\left(H_{1} \frac{\partial \overline{v_{1}^{\prime} \Psi_{1}^{\prime}}}{\partial y}+H_{2} \frac{\partial \overline{v_{2}^{\prime} \Psi_{2}^{\prime}}}{\partial y}+H_{2} \frac{\partial \overline{v_{1}^{\prime} \Psi_{1}^{\prime} q_{1}^{\prime}}}{\partial y}+H_{2} \frac{\partial \overline{v_{2}^{\prime} \Psi_{2}^{\prime} q_{2}^{\prime}}}{\partial y}\right)=-H_{1} \overline{F_{1}^{\prime} \Psi_{1}^{\prime}}-H_{2} \overline{F_{2}^{\prime} \Psi_{2}^{\prime}} \\
& \left.\quad-H_{2} \epsilon \overline{\left(u_{2}^{\prime}\right)^{2}}+\overline{\left(v_{2}^{\prime}\right)^{2}}\right]-H_{2} \epsilon \frac{\partial \overline{\Psi_{2}^{\prime} u_{2}^{\prime}}}{\partial y}
\end{aligned}
$$

where $K_{1}^{\text {ed }}, K_{2}^{\text {ed }}$, and $P^{\text {ed }}$ are the eddy kinetic energy in the upper and lower layers and the eddy available potential energy, respectively. If (39) is integrated over the channel width, all the "divergent" (with the operator $\partial / \partial y$ ) terms disappear, giving (Ivchenko et al. 1997, 2014) 


$$
\begin{aligned}
\frac{\partial}{\partial t} \int_{0}^{L}\left(K_{1}^{\mathrm{ed}}+K_{2}^{\mathrm{ed}}+P^{\mathrm{ed}}\right) d y=0= & -\int_{0}^{L}\left(H_{1} \overline{u_{1}} \overline{v_{1}^{\prime} q_{1}^{\prime}}+H_{2} \overline{u_{2}} \overline{v_{2}^{\prime} q_{2}^{\prime}}\right) d y \\
& \left.-\int_{0}^{L}\left\{H_{1} \overline{F_{1}^{\prime} \Psi_{1}^{\prime}}+H_{2} \overline{F_{2}^{\prime} \Psi_{2}^{\prime}}+H_{2} \epsilon \overline{\left(u_{2}^{\prime}\right)^{2}}+\overline{\left(v_{2}^{\prime}\right)^{2}}\right]\right\} d y
\end{aligned}
$$

The first term on the right-hand side is the generation of eddy mechanical energy by baroclinic and barotropic instabilities. The right-hand side contains dissipative terms due to lateral and bottom friction. For steadystate solutions, positive eddy mechanical energy could occur only if the generation term is negative:

$$
\int_{0}^{L}\left(H_{1} \overline{u_{1}} \overline{v_{1}^{\prime} q_{1}^{\prime}}+H_{2} \overline{u_{2}} \overline{v_{2}^{\prime} q_{2}^{\prime}}\right) d y<0 .
$$

Using (35) the inequality expression (41) can be rewritten in the form

$$
\int_{0}^{L}\left(K_{1} H_{1}{\overline{u_{1}}}^{\mathrm{PV}} \frac{\partial \overline{q_{1}}}{\partial y}+K_{2} H_{2}{\overline{u_{2}}}^{\mathrm{PV}} \frac{\partial \overline{q_{2}}}{\partial y}\right) d y>0 .
$$

The integration of the time- and zonal-mean potential vorticity equations (31) and (32) in the meridional direction for a steady regime, disregarding horizontal friction, yields

$$
\begin{aligned}
& \overline{v_{1}^{\prime} q_{1}^{\prime}}=-\frac{1}{H_{1}} \tau_{x}, \quad \text { and } \\
& \overline{v_{2}^{\prime} q_{2}^{\prime}}=\epsilon \bar{u}_{2}^{\mathrm{PV}},
\end{aligned}
$$

where the constants of integration in (43)-(44) are set to zero because the eddy fluxes, wind stress, and zonalmean velocity are enforced to zero on the solid boundaries in our solutions (Marshall 1981; Ivchenko et al. 2013). Substitution of (35) in (43)-(44) gives

$$
\begin{aligned}
& K_{1} \frac{\partial \overline{q_{1}}}{\partial y}=\frac{1}{H_{1}} \tau_{x}, \quad \text { and } \\
& K_{2} \frac{\partial \overline{q_{2}}}{\partial y}=-\epsilon \bar{u}_{2}^{\mathrm{PV}} .
\end{aligned}
$$

To solve (45)-(46), we need to know the coefficients $K_{1}$ and $K_{2}$. Their values can be found from some additional closures based on equations for eddy kinetic energy (Eden and Greatbatch 2008; Eden 2010) or enstrophy (Ivchenko 1984, 1987) or can be prescribed. We will prescribe constant values (to facilitate analytical solutions) or meridional dependencies based on results of fine-resolution numerical experiments.

Finding an analytical solution for velocities in the case of meridionally varying $\mathrm{CPV}$ is straightforward if the relative vorticity in the QPV can be disregarded compared with the planetary vorticity or the "stretching" term. In that case from (45) and (46), we can write

$$
\begin{aligned}
& \left(\bar{u}_{1}^{\mathrm{PV}}-\bar{u}_{2}^{\mathrm{PV}}\right)=\frac{g^{\prime}}{K_{1} f_{0}^{2}} \tau_{x}-\frac{\beta g^{\prime} H_{1}}{f_{0}^{2}}, \text { and } \\
& \left(\bar{u}_{1}^{\mathrm{PV}}-\bar{u}_{2}^{\mathrm{PV}}\right)=\frac{g^{\prime} H_{2}}{f_{0}^{2}}\left(\beta+\frac{\epsilon}{K_{2}} \bar{u}_{2}^{\mathrm{PV}}\right),
\end{aligned}
$$

which immediately gives for velocities

$$
\begin{aligned}
& \bar{u}_{1}^{\mathrm{PV}}=\left(\frac{\Theta}{H_{2} \epsilon}+\frac{g^{\prime}}{K_{1} f_{0}^{2}}\right) \tau_{x}-\frac{K_{2} \beta H}{H_{2} \epsilon}-\frac{\beta g^{\prime} H_{1}}{f_{0}^{2}}, \text { and } \\
& \bar{u}_{2}^{\mathrm{PV}}=\frac{\Theta}{H_{2} \epsilon} \tau_{x}-\frac{K_{2} \beta H}{H_{2} \epsilon} .
\end{aligned}
$$

Here, $\Theta=K_{2} / K_{1}$.

These solutions will be further compared to the solutions obtained for the $\mathrm{TH}$ parameterization, in which the Reynolds stresses are neglected. To make the analytical treatment in this case simpler, we resort to further simplification, assuming the QPV diffusivities to be constant.

\section{b. Constant values of $C P V$}

If the CPV are constant, the solution derived by Ivchenko et al. (1997, 2013) can be used. The derivation relies on the asymptotic expansion in the small parameter $\gamma=L_{R} / L$, the ratio of the internal Rossby radius $L_{R}$ to the width of the channel. Our equations contain this parameter in the terms with the highest derivatives coming from the relative vorticity. To satisfy the boundary condition of no eddy flux at lateral walls, we set the coefficients $K_{1}$ and $K_{2}$ linearly to zero in thin layers adjacent to the walls.

The solutions for the mean zonal velocities outside the two thin boundary layers near the walls are

$$
\begin{aligned}
& \bar{u}_{1}^{\mathrm{PV}}=u_{c}\left[\left(\frac{\operatorname{Re} \delta_{1}}{\pi}+\frac{\operatorname{Re} \delta_{1}}{D \delta_{2} \pi}\right) \sin \left(\frac{\pi y}{L}\right)-\delta_{1}-\frac{1}{D \delta_{2}}\right]+O\left(\gamma^{2}\right) \\
& \bar{u}_{2}^{\mathrm{PV}}=u_{c}\left[\left(\frac{\operatorname{Re} \delta_{1}}{D \delta_{2} \pi}\right) \sin \left(\frac{\pi y}{L}\right)-\frac{1}{D \delta_{2}}\right]+O\left(\gamma^{2}\right)
\end{aligned}
$$

where $u_{c}=g^{\prime} \beta H / f_{0}^{2}, \delta_{i}=H_{i} / H, \operatorname{Re}=u_{s} L / K_{1}, u_{s}=\left(\pi \tau_{0}\right) /$ $\left(H_{1} \beta L\right)$, and $D=\left(\epsilon u_{c}\right) /\left(\beta K_{2}\right)$. The solutions for PV 
parameterization here are expressed in terms of dimensionless parameters as in Ivchenko et al. (2013). They can be easily rewritten in terms of dimensional parameters.

The next important step lies in applying the Bretherton theorem [(38)]. From this theorem, it is straightforward to demonstrate that $\Theta=\left(K_{2} / K_{1}\right)>1$ (Marshall 1981; Ivchenko et al. 1997) and that

$$
\Theta=\frac{2 \operatorname{Re} \delta_{1}}{2 \operatorname{Re} \delta_{1}-\pi^{2}} .
$$

\section{c. Variable $C P V$}

Eddy-resolving numerical experiments demonstrate that the CPV in a zonal flat bottom channel have a double-peak distribution: the highest values of coefficients occur at the flanks of the flow and reach a local minimum at the center of the channel where the external forcing and jet velocity attain maximum (McWilliams and Chow 1981; Wolff et al. 1991; Olbers 2005), which indicates that zonal jets can acts as barriers for meridional turbulent exchange (Dritschel and McIntyre 2008; Eden 2010). We model such distribution in both layers (see Fig. 1), taking

$$
K_{i}=A_{i} F_{\mathrm{PV}}(y), \quad i=1,2,
$$

where $A_{\mathrm{PV}}=$ const are the amplitudes and $F_{\mathrm{PV}}$ is the normalized profile, $1 / L \int_{0}^{L} F_{\mathrm{PV}} d y=1$. The meridional coordinate in all figures is made dimensionless, $y^{*}=$ $L^{-1} y$.

Given the profile $F_{\mathrm{PV}}$ and prescribing $A_{2}$, the value of $A_{1}$ (i.e., $K_{1}$ ) can easily be found from the Bretherton theorem, which can be written as

$$
A_{1}=\frac{A_{2} \int_{0}^{L} \tau_{x} d y}{\int_{0}^{L} \tau_{x} d y+A_{2} \beta H L} .
$$

\section{Comparison of solutions for $\mathrm{TH}$ parameterization without the contribution of the Reynolds stresses and solutions for PV parameterization}

\section{a. Constraints on the coefficients}

\section{1) TH PARAMETERIZATION}

There is only a trivial constraint $K_{H}>0$ that ensures dissipation (transfer to eddies) of the mean available potential energy.

\section{2) PV PARAMETERIZATION}

The coefficients $K_{1}$ and $K_{2}$ must obey the Bretherton theorem [(38)] and the energy inequality [(42)]. Other constraints may also exist, as hinted by Marshall et al. (2012). They are related to the eddy energy, which is not explicitly modeled here. The Bretherton theorem for the constant (outside the wall boundary layers) CPV can be written in the form

$$
D=\delta_{1} \alpha_{B} \alpha_{U} \operatorname{Re}-0.5 \pi^{2} \alpha_{B} \alpha_{U},
$$

where

$$
\alpha_{B}=\frac{L_{R} u_{c}}{\delta_{1} u_{s} L}
$$

and

$$
\alpha_{U}=\frac{\epsilon}{\beta L_{R}} .
$$

These parameters express the ratio of the stretching term to wind stress in the meridional QPV gradient in the upper layer $\left(\alpha_{B}\right)$ and of the time scale of zonal baroclinic Rossby wave to that of dissipation by bottom friction $\left(\alpha_{U}\right)$. It is easy to show that $K_{2}>K_{1}$ [see (53) and Fig. 2], which is the condition for baroclinic instability (Marshall 1981). The parameters Re and $D$ are inversely proportional to $K_{1}$ and $K_{2}$, respectively, and therefore (56) provides a link between CPV in upper and lower layers. If $K_{1}\left(K_{2}\right)$ is prescribed, then the value of $K_{2}\left(K_{1}\right)$ must comply with (56).

One more constraint is based on the mechanical energy transfer term [(42)] (Ivchenko et al. 1997, 2014). Since in the eddying regime a steady state may exist only for a positive mechanical energy flux from the mean flow to eddies, the following restriction should be observed (Ivchenko et al. 2014):

$$
\begin{aligned}
G_{\mathrm{PV}}= & 3 \delta_{1} \delta_{2} \operatorname{Re} D^{2}+3 \delta_{1} \operatorname{Re} D-12 \delta_{1} \delta_{2} D^{2}-12 D \\
& +24 \delta_{1} \alpha_{B} \alpha_{U} \operatorname{Re}-6 \pi^{2} \alpha_{B} \alpha_{U}-3 \delta_{1}^{2} \alpha_{B} \alpha_{U} \operatorname{Re}^{2}>0,
\end{aligned}
$$

where $G_{\mathrm{PV}}$ is the generation of the eddy mechanical energy. This energy inequality (EI) does not allow using the values of $K_{2}$ in excess of the critical value depending on $K_{1}$. In Fig. $2, K_{2}$ lies below the red line; $K_{2}$ is greater than $K_{1}$ so the points must lie above the black line on the green line corresponding to the Bretherton theorem. In physical terms it implies that for baroclinic instability to develop the vertical shear of the zonally averaged zonal velocity must be higher than the critical value (Marshall 1981; Ivchenko et al. 1997). 


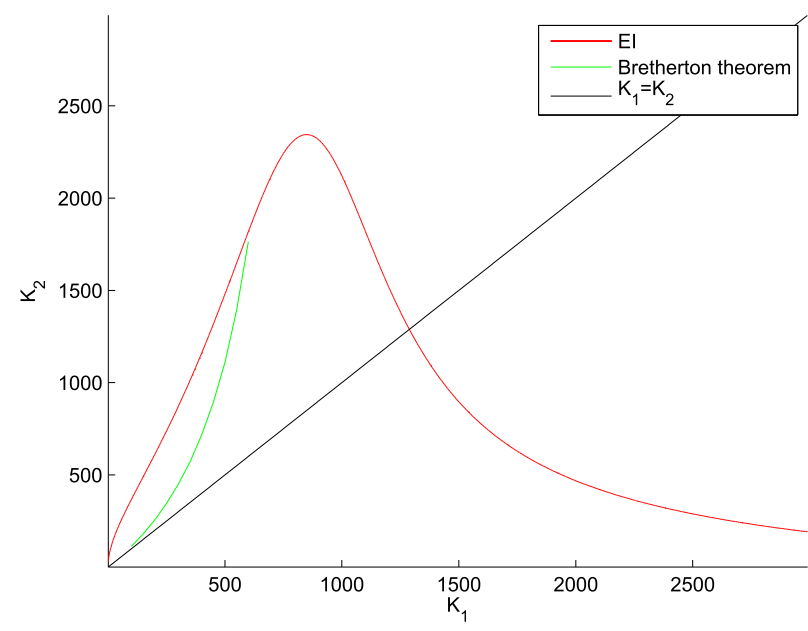

FIG. 2. The space of the allowable coefficients $K_{1}$ and $K_{2}$. The admissible values of CPV must lie beneath the red line (EI) and must lie on the green line (Bretherton theorem).

\section{b. Zonal velocity}

\section{1) TH PARAMETERIZATION}

The zonally averaged zonal velocity [(25)] in the lower layer is proportional to the wind stress and independent of the coefficient $K_{H}$ (Fig. 3). The velocity in the upperlayer [(29)] is also proportional to the wind stress [(29)], but retains the dependence on $K_{H}$ (Fig. 3).

\section{2) PV PARAMETERIZATION}

In the case of uniform $K_{i}$ (outside thin wall boundary layers), the meridional profile of zonally averaged zonal velocity in the lower layer comprises two terms. The first one is similar to the expression for the zonally averaged zonal velocity in the case of TH parameterization [proportional to the wind stress and inversely proportional to the $\left.\epsilon \mathrm{H}_{2}(50)\right]$. The major difference between the TH and PV solutions is the presence of $\Theta=K_{2} / K_{1}$ that must obey the Bretherton theorem. So, the zonally averaged zonal velocity profile is modified (as compared to the $\mathrm{TH}$ case) in the meridional direction to agree with the momentum conservation (see Fig. 3). The second term is $\left(\Theta \beta K_{1}\right) /\left(\epsilon \delta_{2}\right)$, which complies with the momentum conservation and depends on the planetary vorticity gradient, bottom viscosity, geometry parameter $\delta_{2}$, and coefficient $K_{1}$. It is negative and constant for the prescribed constant coefficients $K_{1}$ and $K_{2}$. Importantly,

$$
\int_{0}^{L} \bar{u}_{2}^{\mathrm{PV}} d y=\frac{2 L \tau_{0}}{\pi H_{2} \epsilon}
$$

These expressions are equal for both parameterizations. It is not surprising because there should be the same balance in the steady state for the same forcing.
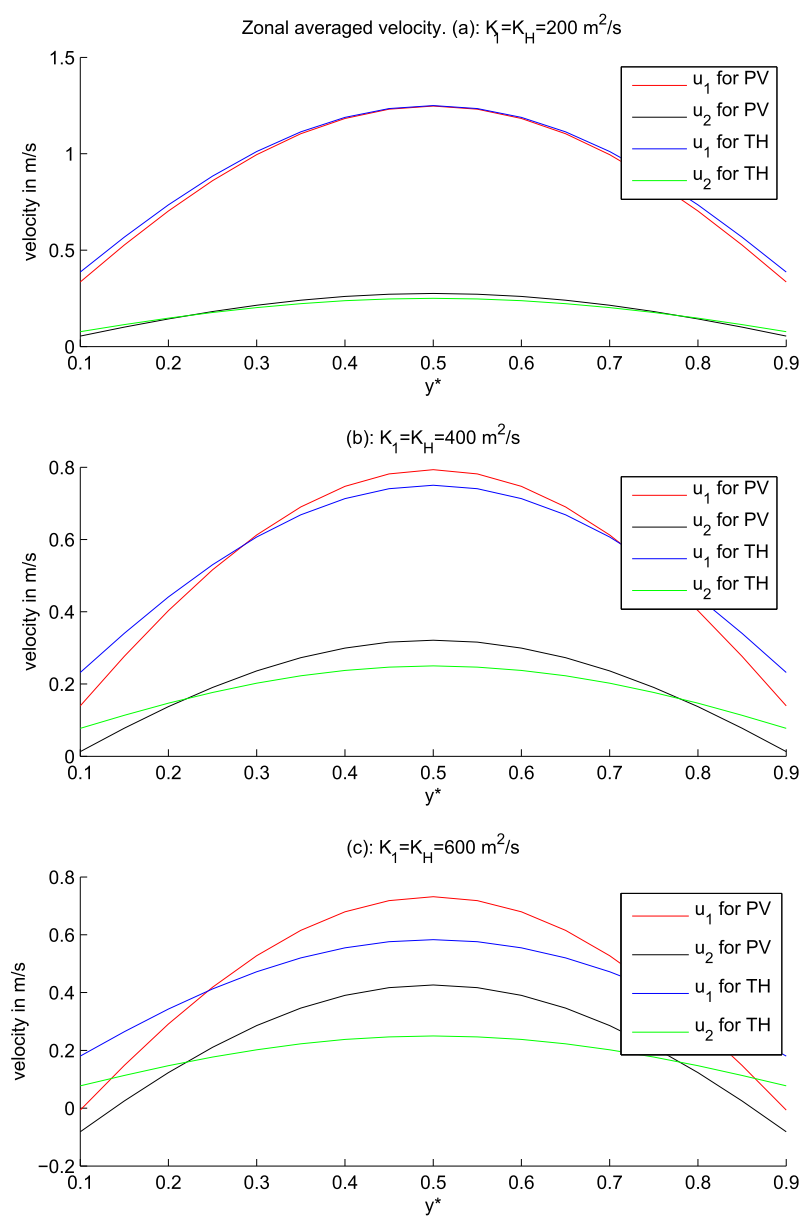

FIG. 3. Zonally averaged zonal velocities; the CPV and CT are constant.

The zonally averaged zonal velocity profile in the upper layer is the sum of the lower-layer velocity and two additional terms. The first one is proportional to the wind stress and is equal to the corresponding term for the thickness parameterization up to exchange of $K_{H}$ and $K_{1}$. The second term is $\delta_{1} g^{\prime} \beta H / f_{0}^{2}$.

The zonally averaged zonal velocity profiles in each of the layers for $\mathrm{PV}$ and $\mathrm{TH}$ parameterizations are close to each other for small values of CPV and CT if $K_{1}=K_{H}$ (Fig. 3). We call $K_{1}$ "small" if

$$
\operatorname{Re} \gg \pi^{2} / 2 \delta_{1},
$$

( $\mathrm{Re}$ is inversely proportional to $K_{1}$ ), which implies accounting for (53):

$$
\Theta \approx 1
$$

It is straightforward to demonstrate that the second term in the expression for the upper-layer zonally averaged zonal velocity for the PV parameterization 
[(49)] dominates over the other terms for small $K_{1}$, so that

$$
\bar{u}_{1}^{\mathrm{PV}} \approx \frac{g^{\prime}}{f_{0}^{2} K_{1}} \tau_{x}
$$

The second term in the expression for the zonally averaged zonal velocity in the upper layer for TH parameterization [(29)] dominates over the first term for small $K_{H}$, and therefore the zonally averaged zonal velocity profiles there are close to each other for PV and $\mathrm{TH}$ parameterization for small but equal diffusivities $K_{1}=$ $K_{H}$ (see Fig. 3a).

In the lower layer, the zonally averaged zonal velocity in the PV parameterization case is determined by the first term in (50) for small CPV because the second term is proportional to $K_{2}$. Since $\Theta \approx 1$, the velocities for the lower layers for $\mathrm{PV}$ and $\mathrm{TH}$ parameterization are close to each other for small but equal $K_{1}=K_{H}$ (see Fig. 3a). Increasing coefficient $K_{1}$ (and correspondingly $K_{H}$ ) from 200 (in Fig. 3a) to 400 and $600 \mathrm{~m}^{2} \mathrm{~s}^{-1}$ increases the difference between the velocity profiles (Figs. 3b,c). Note that we cannot investigate the asymptotic limit of large CPV and CT because $K_{1}$ must be less than the critical value set by momentum and energy conservation (Fig. 2).

\section{c. Production of eddy mechanical energy}

\section{1) TH PARAMETERIZATION}

The transfer of total mean mechanical energy to eddies is given by $\Pi=-K_{H}\left(\partial \bar{h}_{3 / 2} / \partial y\right)^{2}$, corresponding to baroclinic instability. It is negative (i.e., baroclinic instability is maintained for any vertical shear of the zonal-mean velocities). It can be computed in the case considered to give

$$
\Pi=-\frac{\tau_{0}^{2} g^{\prime} L}{2 f_{0}^{2} K_{H}}
$$

for $K_{H}=$ const. It is inversely proportional to $K_{H}$ and strongly increases when $K_{H}$ decreases (Fig. 4).

\section{2) PV PARAMETERIZATION}

The production of eddy energy has already been discussed above.

\section{d. Zonal transport}

\section{1) TH PARAMETERIZATION}

The zonal transport $T$ can be found by multiplying velocities (25) and (29) with the corresponding layer thicknesses $H_{i}$, summing them and integrating over the channel width:
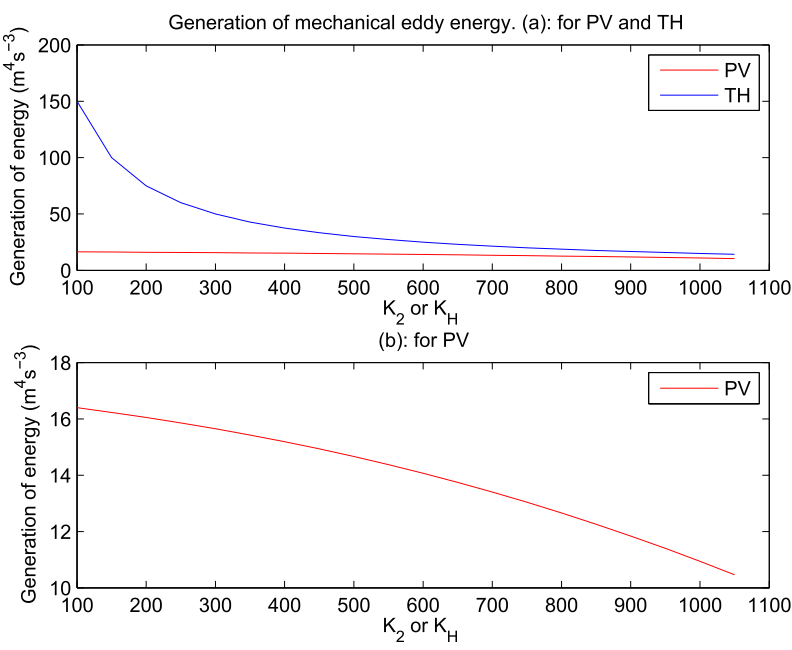

FIG. 4. Generation of meridionally and vertically integrated mechanical eddy energy for (top) PV and TH parameterization and for (bottom) PV parameterization with different scaling.

$$
T=\frac{2 H \tau_{0} L}{\pi \epsilon H_{2}}+\frac{2 g^{\prime} H_{1} \tau_{0} L}{f_{0}^{2} K_{H} \pi} .
$$

It strongly increases if $K_{H}$ is reduced because of the term inversely proportional to $K_{H}$ (see Fig. 5). For large $K_{H}$ it approaches the value

$$
\lim _{K_{H} \rightarrow \infty} T=\frac{2 H \tau_{0} L}{\pi \epsilon H_{2}} .
$$

\section{2) PV PARAMETERIZATION}

The zonal transport $T$ can be found by multiplying velocities (51) and (52) with the corresponding layer thicknesses $H_{i}$, summing them and integrating over the channel width:

$$
T=L H u_{c}\left(\frac{2 \delta_{1}^{2} \mathrm{Re}}{\pi^{2}}+\frac{2 \delta_{1} \mathrm{Re}}{\delta_{2} \pi^{2} D}-\delta_{1}^{2}-\frac{1}{\delta_{2} D}\right) .
$$

The behavior of zonal transports for PV and TH parameterization appears similar: the transports strongly increase if $K_{i}$ and $K_{H}$ are reduced, but show no changes for large enough diffusivities. An interesting question is how large should be the differences between the coefficients $K_{i}$ and $K_{H}$ if we want to get the same zonal transport. If we set $K_{1}=K_{H}$ (and calculate $K_{2}$ from the Bretherton theorem), the transports in $\mathrm{PV}$ and $\mathrm{TH}$ parameterization will be close to each other (see Fig. 5a). However, if we set $K_{2}=K_{H}$, very different values of diffusivities will be required to warrant the same transports (Fig. 5b). 

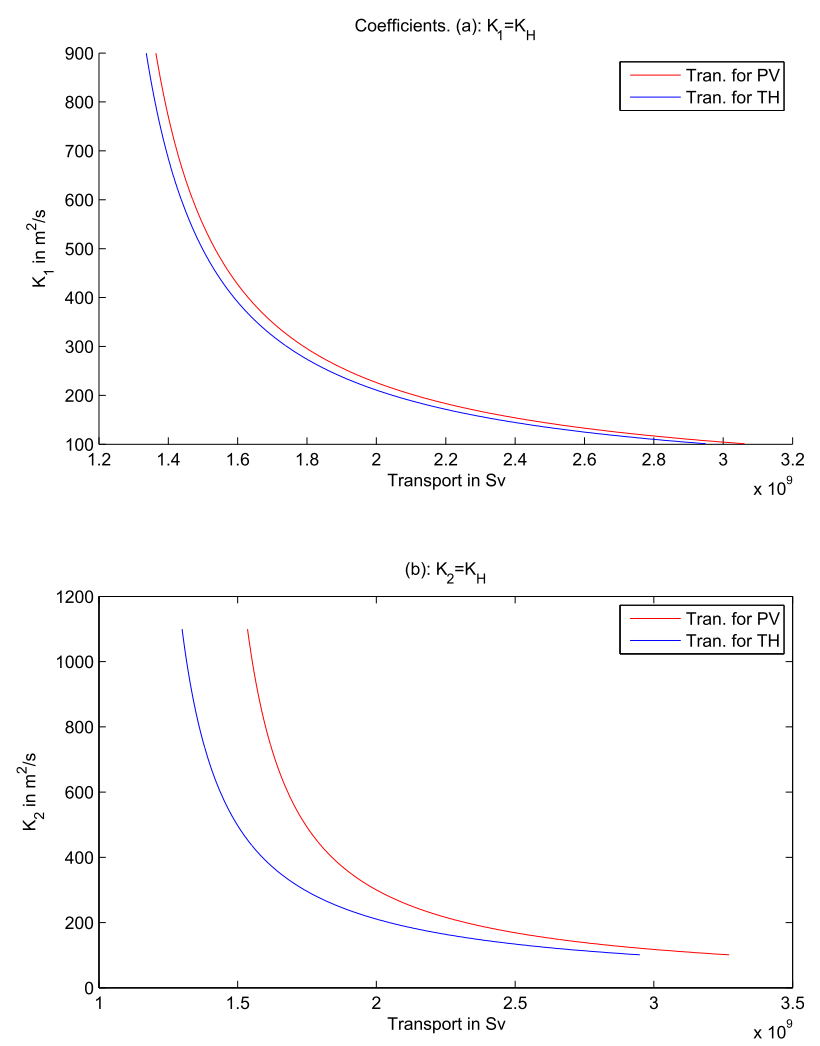

FIG. 5. Links between coefficients and zonal transport. (a) Relation between $K_{1}$ and transport, $K_{H}=K_{1}$. (b) Relation between $K_{2}$ and transport, $K_{H}=K_{2}$.

\section{e. Variable coefficients}

The zonal velocities are strongly dependent on the coefficients $K_{1}, K_{2}$ and $K_{H}$. If diffusivities are small, the velocities in the lower layer show almost identical sinusoidal behavior, but in the upper layer, the PV parameterization results in stronger flows (see Fig. 6a). As $A_{2}$ increases, the difference between $\bar{u}_{2}$ for the PV and TH parameterization increases (Figs. $6 \mathrm{~b}, \mathrm{c}$ ), since planetary vorticity plays a defining role. The velocity amplitudes in the upper layers decrease for both parameterizations with increase in $K_{i}$ and $K_{H}$.

Transport calculated for the PV and TH parameterizations is similar if we set $K_{2}=K_{H}$ (Fig. 7), however, with higher values for the PV parameterization. It is strongly nonlinear for small values of coefficients.

\section{Parameterization of eddy PV fluxes based on the eddy enstrophy budget}

As we have seen, the zonally averaged zonal velocity profiles strongly depend on the meridional profiles of the CPV. The CPV need to be parameterized either directly in terms of mean fields or linked with the dynamics of other parameterized fields such as eddy kinetic energy or enstrophy. Many studies prefer the eddy kinetic energy (Eden and Greatbatch 2008; Eden 2010). However, in the case of jet flows, the eddy kinetic energy reaches a maximum at the jet centers, while the CPV has a local minimum there (McWilliams and Chow 1981). Reconciling these behaviors requires special hypotheses on the mixing length scale.

An alternative view can be based on the approach that deals with the balance of the eddy quasigeostrophic potential enstrophy (EQPE). To derive the equation on the EQPE in the upper layer, we multiply (6) by the eddy component of QPV and average the result zonally and over time:

$$
\overline{q_{1}^{\prime} \frac{\partial q_{1}}{\partial t}}+\overline{q_{1}^{\prime} J\left(\Psi_{1}, q_{1}\right)}=\frac{1}{H_{1}} \overline{q_{1}^{\prime} \operatorname{curl}_{z} \tau}+\overline{F_{1}^{\prime} q_{1}^{\prime}} .
$$

The first term on the lhs is the time derivative of the upper-layer EQPE:

$$
\overline{q_{1}^{\prime} \frac{\partial q_{1}}{\partial t}}=\frac{\partial}{\partial t}\left(\frac{1}{2} \overline{q_{1}^{\prime 2}}\right)=0 .
$$

The first term on the rhs of (68) is zero if the wind stress is steady. The nonlinear advection term can be rearranged as

$$
\begin{aligned}
\overline{q_{1}^{\prime} J\left(\Psi_{1}, q_{1}\right)} & =\overline{q_{1}^{\prime} v_{1}^{\prime}\left(\frac{\partial \overline{q_{1}}}{\partial y}+\frac{\partial q_{1}^{\prime}}{\partial y}\right)}+\overline{q_{1}^{\prime}\left(\overline{u_{1}}+u_{1}^{\prime}\right) \frac{\partial q_{1}^{\prime}}{\partial x}} \\
& =\overline{q_{1}^{\prime} v_{1}^{\prime}} \frac{\partial \overline{q_{1}}}{\partial y}+\frac{1}{2} \frac{\partial \overline{v_{1}^{\prime} q_{1}^{\prime 2}}}{\partial y}-\frac{1}{2} \overline{q_{1}^{\prime 2}\left(\frac{\partial v_{1}^{\prime}}{\partial y}+\frac{\partial u_{1}^{\prime}}{\partial x}\right)} .
\end{aligned}
$$

The last term on the rhs of (70) is zero because the horizontal divergence of quasigeostrophic velocity is zero. On substitution of (69) and (70) into (68), it becomes

$\frac{\partial}{\partial t}\left(\frac{1}{2} \overline{q_{1}^{\prime 2}}\right)=0=-\overline{q_{1}^{\prime} v_{1}^{\prime}} \frac{\partial \overline{q_{1}}}{\partial y}-\frac{1}{2} \frac{\partial \overline{v_{1}^{\prime} q_{1}^{\prime 2}}}{\partial y}+\overline{F_{1}^{\prime} q_{1}^{\prime}}$.

The second term on the rhs of (71) is the eddy redistribution of the EQPE; it disappears after integration over the channel width. The last term represents the dissipation mechanism. The first term on the rhs is the EQPE generation. On substituting (35), it becomes

$$
-\overline{q_{1}^{\prime} v_{1}^{\prime}} \frac{\partial \overline{q_{1}}}{\partial y}=K_{1}\left(\frac{\partial \bar{q}_{i}}{\partial y}\right)^{2} .
$$

The eddy enstrophy production term is proportional to the CPV times the mean PV gradient squared, which 

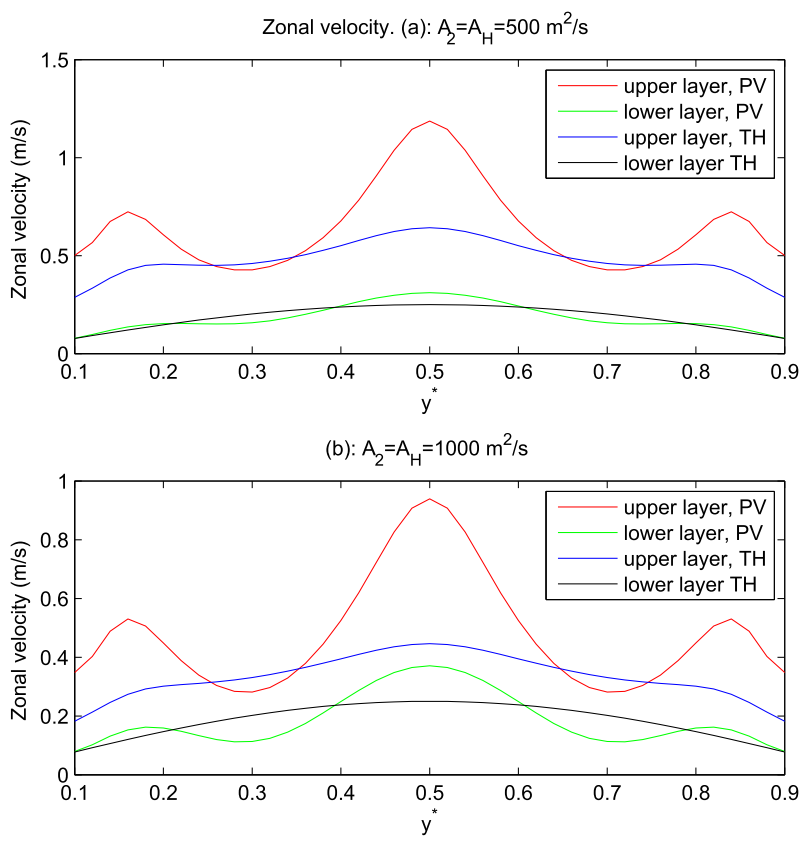

(c): $A_{2}=A_{H}=2000 \mathrm{~m}^{2} / \mathrm{s}$

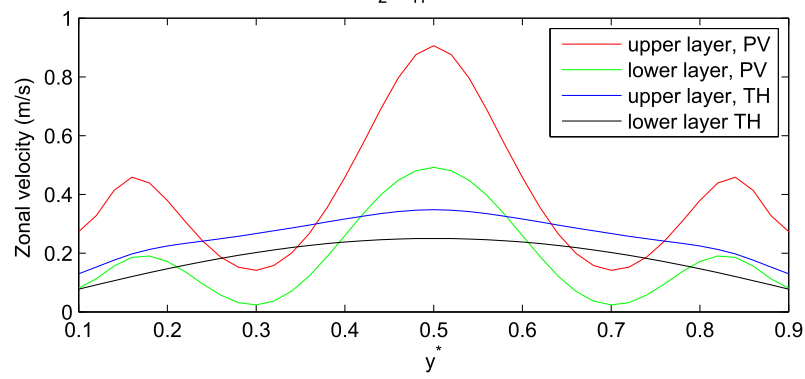

FIG. 6. Zonally averaged zonal velocity in upper and lower layers for PV and TH parameterization: (a) $A_{2}=A_{H}=500 \mathrm{~m}^{2} \mathrm{~s}^{-1}$, (b) $A_{2}=A_{H}=1000 \mathrm{~m}^{2} \mathrm{~s}^{-1}$, and (c) $A_{2}=A_{H}=2000 \mathrm{~m}^{2} \mathrm{~s}^{-1}$.

is compatible with small values of the CPV at the jet centers (McWilliams and Chow 1981). For that reason we prefer assessing the CPV from the EQPE equation. If the dissipation and eddy advection of eddy enstrophy in the EQPE are known or parameterized, the CPV can be found (Ivchenko 1984; Ivchenko et al. 1997):

$$
K_{1}=\frac{\frac{\partial}{\partial y}\left(\frac{1}{2} \overline{v_{1}^{\prime} q_{1}^{\prime 2}}\right)-\overline{F_{1}^{\prime} q_{1}^{\prime}}}{\left(\frac{\partial \overline{q_{1}}}{\partial y}\right)^{2}} .
$$

Numerical experiments demonstrate that in strong baroclinic regimes the sum of eddy redistribution of EQPE and dissipation of EQPE in the numerator in (73) has a strong correlation with the basic parameter of baroclinic instability-the vertical shear of mean zonal velocity $\left(\bar{u}_{1}-\bar{u}_{2}\right)$ (see Fig. 12 in Ivchenko et al. 1997).

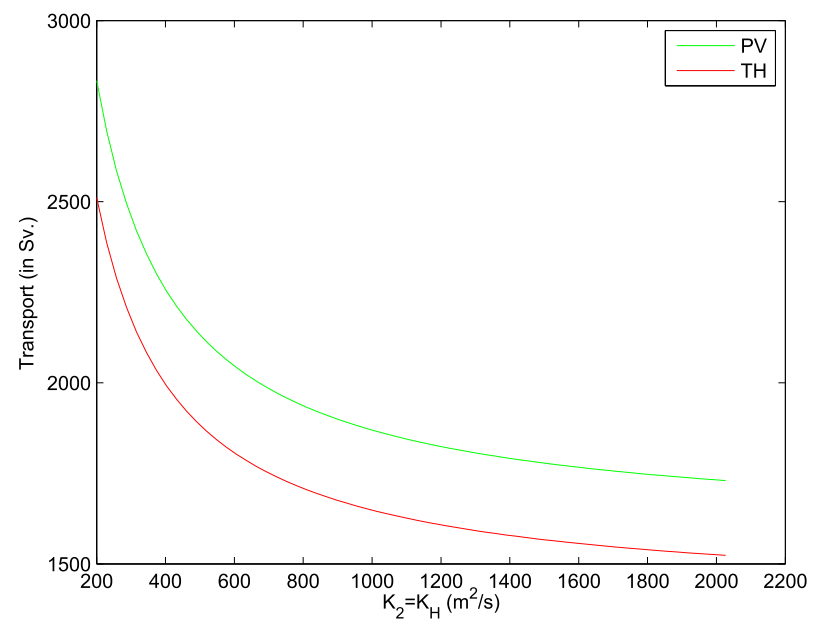

FIG. 7. Zonal transport for PV parameterization and TH parameterization as function of $K_{2}$ and $K_{H}$.

Assuming that the numerator is proportional to the modulus of $\bar{u}_{1}-\bar{u}_{2}$, we can write

$$
K_{1}=\frac{\left|\bar{u}_{1}^{\mathrm{PV}}-\bar{u}_{2}^{\mathrm{PV}}\right|}{\mu_{1}\left(\frac{\partial \overline{q_{1}}}{\partial y}\right)^{2}},
$$

where $\mu_{1}$ is some coefficient $\left(\mathrm{ms}^{2}\right)$. We use the term "strong baroclinic regime" to designate the flows with high values of mean meridional QPV gradients. Marshall (1981) used the CPV proportional to the vertical shear of the mean zonal velocities, which provides satisfactory solution for the weak baroclinic regimes, that is, for small meridional gradients of QPV:

$$
K_{1} \sim\left(\left|\bar{u}_{1}^{\mathrm{PV}}-\bar{u}_{2}^{\mathrm{PV}}\right|\right) .
$$

Such expressions provide the local maximum for the $\mathrm{CPV}$ in the center of the jet. It would be interesting to combine expressions for the CPV in both regimes, that is, strong baroclinic [(74)] and weak baroclinic [(75)], in the general expression. Adding a constant $\mu_{2}\left(\mathrm{~m}^{-1}\right)$ to the denominator we get

$$
K_{1}=\frac{\left|\bar{u}_{1}^{\mathrm{PV}}-\bar{u}_{2}^{\mathrm{PV}}\right|}{\mu_{1}\left(\frac{\partial \overline{q_{1}}}{\partial y}\right)^{2}+\mu_{2}} .
$$

According to this expression, in the strongly baroclinic regime, for instance around the jet center, the CPV is inversely proportional to the mean meridional QPV gradient squared, which ensures a local minimum in the CPV. In the regions where the gradients of mean QPV 
are small, the CPV is proportional to vertical shear of zonally averaged zonal velocity. If we substitute expressions for $\bar{u}_{1}^{\mathrm{PV}}$ and $\bar{u}_{2}^{\mathrm{PV}}[(49),(50)]$ and for the meridionalmean QPV gradient into (76), we obtain the quadratic equation on $K_{1}$ :

$$
K_{1}^{2}+\frac{g^{\prime} \beta H_{1}}{\mu_{2} f_{0}^{2}} K_{1}+\left(\frac{\mu_{1} \tau_{x}^{2}}{\mu_{2} H_{1}^{2}}-\frac{g^{\prime} \tau_{x}}{f_{0}^{2} \mu_{2}}\right)=0,
$$

with the solution

$$
K_{1}=-\frac{g^{\prime} \beta H_{1}}{2 f_{0}^{2} \mu_{2}}+\left(2 f_{0}^{2} H_{1} \mu_{2}\right)^{-1} \sqrt{g^{\prime 2} \beta^{2} H_{1}^{4}-4 f_{0}^{4} \tau_{x}^{2} \mu_{1} \mu_{2}+4 f_{0}^{2} g^{\prime} \tau_{x} H_{1}^{2} \mu_{2}} .
$$

The expression under the square root of (78) must be positive. This condition restricts the coefficient $\mu_{1}$ :

$$
\mu_{1}<\frac{g^{\prime} \beta^{2} H_{1}^{4}}{4 \mu_{2} f_{0}^{4} \tau_{x}^{2}}+\frac{g^{\prime} H_{1}^{2}}{f_{0}^{2} \tau_{x}} .
$$

Since $K_{1}$ is positive, the first term in (78) must be smaller than the second term, which gives

$$
\mu_{1}<\frac{g^{\prime} H_{1}^{2}}{f_{0}^{2} \tau_{x}}
$$

The rhs of (80) equals the second term on the rhs of (79). Because the first term in (79) is positive, condition (80) is stronger than (79). The rhs of (80) is inversely proportional to the wind profile $\sin (\pi y / L)$ and therefore $\mu_{1}$ is most constrained at the channel center $y=L / 2$. For the standard set of parameters, this gives

$$
\mu_{1}<2 \times 10^{16} \mathrm{~m} \mathrm{~s}^{2} .
$$

The coefficient $K_{1}$ is plotted in Fig. 8 as a function of the meridional dimensionless coordinate for various values of parameters $\mu_{1}$ and $\mu_{2}$. The respective curves attain either local maxima (red line in Fig. 8) or local minima (other lines) at the center of the channel. The maxima correspond to weak baroclinic regimes with smooth meridional profiles of the zonally mean zonal velocity, and minima correspond to strong baroclinic regimes with the intensification of zonally mean zonal velocity with respect to the sinusoidal profile of wind stress forcing (see Figs. 9a,b). The profiles of zonally mean zonal velocity do not show strong sensitivity to the coefficient $\mu_{2}$, which could be called the "coefficient of weak baroclinic regime" (see Fig. 9a). They are more sensitive to the parameter $\mu_{1}$ (we call it the "coefficient of strong baroclinic regime") (Fig. 9b), which affects the maximum values at the jet core. It is interesting and important that the total transport in all cases remains almost constant. For the three sets of parameters in the upper and lower panels of Fig. 9, the transports vary around 1700 Sverdrups $\left(\mathrm{Sv} ; 1 \mathrm{~Sv} \equiv 10^{6} \mathrm{~m}^{3} \mathrm{~s}^{-1}\right)$ within $1.5 \%$. The parameterized fluxes redistribute zonal momentum, intensifying the jet, but do not strongly influence the zonal transport.

The zonal channel flows have equivalent-barotropic structure, similar to that of the Antarctic Circumpolar Current (ACC; Killworth 1992; Krupitsky et al. 1996), which allows us to employ similar CPV profiles in both layers with different amplitudes.

\section{The Reynolds stresses and the TH parameterization}

The solutions for zonal velocities for the TH parameterizations contain the Reynolds stresses divergence, that is, the last two terms in (29) and the last term in (25). A question naturally arises: would it be possible to approximate with these solutions the PV solutions [(49)(50)] by taking a special distribution of $K_{H}$ ? In other words, is it possible by special choice of $K_{H}$ to introduce in the $\mathrm{TH}$ solutions the effect of the Reynolds stress divergence? In section 5 , we demonstrate that for constant values of $K_{i}$ and $K_{H}$ and for small $K_{1}$ the solutions for zonal velocities for TH parameterization are close to PV solutions. Similar situations occur for variable coefficients, as well. If choosing $K_{H}=K_{1}$ from the PV parameterization, (29) and (25) can be rewritten omitting the Reynolds stresses and introducing $K_{1}$ instead of $K_{H}$ :

$$
\begin{aligned}
& \bar{u}_{1}^{\mathrm{TH}}=\left(\frac{1}{\epsilon H_{2}}+\frac{g^{\prime}}{f_{0}^{2} K_{1}}\right) \tau_{x}, \text { and } \\
& \bar{u}_{2}^{\mathrm{TH}}=\frac{1}{\epsilon H_{2}} \tau_{x} .
\end{aligned}
$$

If the second term in the denominator of (55) is much smaller than the first one, that is,

$$
A_{2} \ll \frac{\int_{0}^{L} \tau_{x} d y}{\beta H L}
$$

then it leads to $\Theta \approx 1$. For small $K_{1}$, the second and the third terms in (49) will be much smaller than 

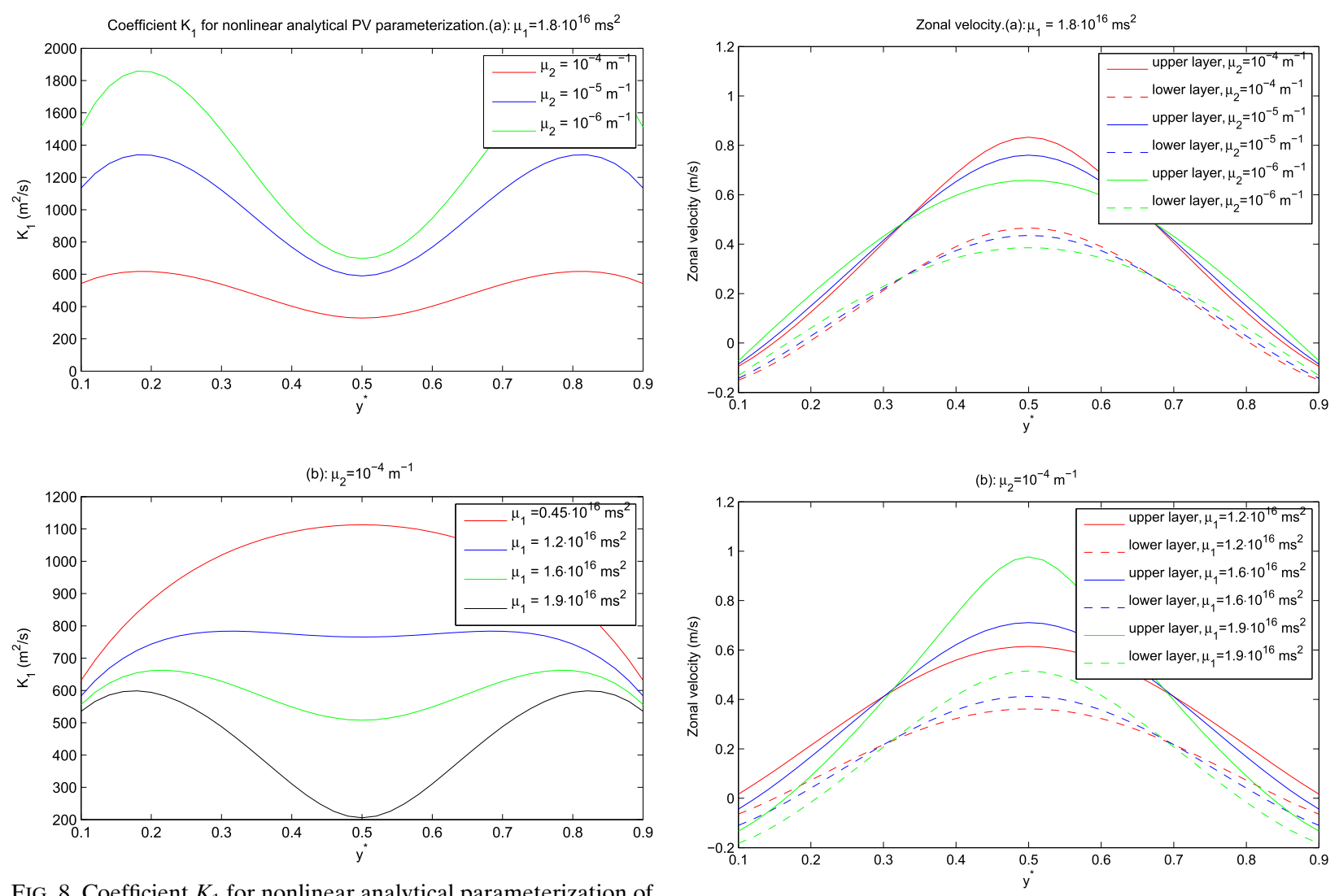

FIG. 8. Coefficient $K_{1}$ for nonlinear analytical parameterization of PV: (a) $\mu_{1}=1.8 \times 10^{16} \mathrm{~m} \mathrm{~s}^{2}$ and (b) $\mu_{2}=10^{-4} \mathrm{~m}^{-1}$.

$$
\begin{aligned}
& \frac{K_{2} \beta H}{H_{2} \epsilon} \ll \frac{g^{\prime}}{K_{1} f_{0}^{2}} \tau_{x}, \quad \text { and } \\
& \frac{\beta g^{\prime} H_{1}}{f_{0}^{2}} \ll \frac{g^{\prime}}{K_{1} f_{0}^{2}} \tau_{x} .
\end{aligned}
$$

Similarly,

$$
\frac{K_{2} \beta H}{H_{2} E} \ll \frac{\Theta}{H_{2} E} \tau_{x}
$$

This means that for small enough values of coefficients, the solutions of TH and PV parameterizations will be close to each other if we use values of coefficient $K_{1}$ instead of $K_{H}$. Relatedly, using the TH parameterization with the diffusivity coefficient obtained from the PV parameterization allows one to describe the effect of the Reynolds stresses, which gives much sharper zonal velocity profiles.

\section{Summary and discussion}

Any parameterization of eddy fluxes must be based on correct physics. Physical arguments suggest that diffusive

FIG. 9. Zonally averaged zonal velocity for nonlinear analytical
parameterization of PV: (a) $\mu_{1}=1.8 \times 10^{16} \mathrm{~m} \mathrm{~s}^{2}$ and (b) $\mu_{2}=$ $10^{-4} \mathrm{~m}^{-1}$.

parameterizations can be applied only to conserved variables. Momentum is not a conserved variable because of the presence of the pressure gradient, and the parameterization of the Reynolds stresses still presents a great challenge. Commonly used harmonic/biharmonic viscosity operators fail to provide an adequate parameterization of eddy fluxes of momentum. For example, the eddy kinetic energy can in some cases be transferred to the mean kinetic energy in eddying flows, which a harmonic operator can only do if the viscosity was negative.

The interest to parameterizations of eddy fluxes of potential vorticity stems from the fact that they incorporate the redistribution of both momentum and thickness. Adopting them, one does not need to separately parameterize the Reynolds stresses. Their disadvantage is that the mean $\mathrm{PV}$ is not a variable of the primitive equation models. In spite of that, recent studies propose the ways of using parameterized PV fluxes in such models (Wardle and Marshall 2000; Eden and Greatbatch 2008; Eden 2010; Marshall and Adcroft 2010; Ringler and Gent 2011; Marshall et al. 2012). 
Here, we compared the effects of PV and TH parameterizations on the mean flow driven by wind in a zonally reentrant flat bottom channel. Although the model used by us is a gross oversimplification of realistic situation, it admits analytical treatment or simple analysis, allowing us to learn about the differences caused by the parameterizations. We considered both uniform and variable meridional profiles of diffusivities, prescribing in the latter case the profiles resembling those diagnosed in the eddy-resolving experiments.

For the constant values of the coefficients, the main difference between solutions for the two parameterizations in the lower layer is that mean zonal velocity in the QPV flux parameterization acquires a factor $\Theta=$ $\operatorname{Re} \delta_{1} /\left(2 \operatorname{Re} \delta_{1}-\pi^{2}\right)$, obtained from the momentum conservation law (the Bretherton theorem). In the flat bottom case, $\Theta>1$, and the maximum in the center of the channel is amplified as compared to the case of $\mathrm{TH}$ parameterization. The profiles of zonally mean zonal velocity for $\mathrm{PV}$ and $\mathrm{TH}$ parameterization are close to each other for small values of CPV and CT; however, the difference between velocities in both layers increases as the coefficients are increased.

The major difference between the two approaches relates to the role of constraints. While there are important integral constraints in the PV parameterization case (based on momentum and energy conservation), no such constraints are associated with the $\mathrm{TH}$ parameterization. There is only a trivial restriction that $K_{H}$ be positive. Using the $\mathrm{TH}$ parameterization is thus easier, but the PV parameterization seems to be more complete and allows one to exercise strict control on the conservation of momentum and energy.

The obvious difference between these two parameterizations can be seen when the CPV and CT vary meridionally. According to numerical experiments, the profile of diffusivity in the TH parameterization is almost constant in the center of the jet, whereas the CPV coefficient $K_{i}$ has pronounced local minima there (McWilliams and Chow 1981). These minima represent a kind of PV barrier (Dritschel and McIntyre 2008). Therefore, using such a more realistic distribution of coefficients provides a very different distribution of zonal velocities with strong momentum concentration in the jet center for the PV parameterization, but without momentum intensification for the $\mathrm{TH}$ parameterization. These results demonstrate the importance of introducing the parameterization scheme with varying diffusivities not only for the Southern Ocean, but for other domains with zonal jets as well. Such closures could be based on eddy enstrophy (Ivchenko 1984; Ivchenko et al. 1997), since the generation term is proportional to the product of the CPV with the square of the mean QPV gradient, that is, $K_{i}\left(\partial \overline{q_{i}} / \partial y\right)^{2}$. The profiles of CPV with a local minimum in the jet center allow one to get the zonal momentum intensification by Reynolds stresses (the "negative viscosity" effect) by using positive CPV. This mechanism redistributes the zonal momentum, increasing it in the jets cores and decreasing it on the flanks, which results in only small change of the zonal transport.

Acknowledgments. We thank two anonymous reviewers for useful comments and discussion that led to a greatly improved manuscript.

\section{REFERENCES}

Bretherton, F. S., 1966: Critical layer instability in baroclinic flows. Quart. J. Roy. Meteor. Soc., 92, 325-334, doi:10.1002/ qj.49709239302.

Danabasoglu, G., J. C. McWilliams, and P. R. Gent, 1994: The role of mesoscale tracer transports in the global ocean circulation. Science, 264, 1123-1126, doi:10.1126/science.264.5162.1123.

Danilov, S., and D. Gurarie, 2002: Rhines scale and spectra of the $\beta$-plane turbulence with bottom drag. Phys. Rev. E Stat. Nonlinear Soft Matter Phys., 65, 067301, doi:10.1103/ PhysRevE.65.067301.

Dritschel, D. G., and M. E. McIntyre, 2008: Multiple jets as PV staircases: The Philips effect and the resilience of eddytransport barriers. J. Atmos. Sci., 65, 855-874, doi:10.1175/ 2007JAS2227.1.

Eden, C., 2010: Parameterising meso-scale eddy momentum fluxes based on potential vorticity mixing and a gauge term. Ocean Modell., 32, 58-71, doi:10.1016/j.ocemod.2009.10.008.

_- and R. J. Greatbatch, 2008: Towards a mesoscale eddy closure. Ocean Modell., 20, 223-239, doi:10.1016/j.ocemod.2007.09.002.

Gent, P. R., and J. C. McWilliams, 1990: Isopycnal mixing in ocean circulation models. J. Phys. Oceanogr., 20,150-155, doi:10.1175/ 1520-0485(1990)020<0150:IMIOCM > 2.0.CO;2.

Green, J. S. A., 1970: Transfer properties of the large-scale eddies and the general circulation of the atmosphere. Quart. J. Roy. Meteor. Soc., 96, 157-185, doi:10.1002/qj.49709640802.

Griffies, S. M., 2004: Fundamentals of Ocean Climate Models. Princeton University Press, $518 \mathrm{pp}$.

Harrison, D. E., 1978: On the diffusion parameterization of mesoscale eddy effects from a numerical ocean experiment. J. Phys. Oceanogr., 8, 913-918, doi:10.1175/1520-0485(1978)008<0913: OTDPOM $>2.0 . \mathrm{CO} ; 2$.

Hirst, A. C., and T. J. McDougall, 1996: Deep water properties and surface buoyancy flux as simulated by a z-coordinate model including eddy-induced advection. J. Phys. Oceanogr., 26, 1320-1343, doi:10.1175/1520-0485(1996)026<1320: DWPASB $>2.0 . \mathrm{CO} ; 2$.

Holland, W. R., 1978: The role of mesoscale eddies in the general circulation of the ocean-Numerical experiments using a winddriven quasi-geostrophic model. J. Phys. Oceanogr., 8, 363-392, doi:10.1175/1520-0485(1978)008<0363:TROMEI >2.0.CO;2.

Ivchenko, V. O., 1984: Parameterization of the eddy fluxes of the quasi-geostrophic potential vorticity in zonal flows. Dokl. Akad. Nauk USSR, 277, 972-976.

_ 1987: Influence of bottom topography on the eddy transfer coefficient. Izv. Akad. Nauk USSR, Atmos. Ocean Phys., 23, 200-208. 
— K. J. Richards, and D. P. Stevens, 1996: The dynamics of the Antarctic Circumpolar Current. J. Phys. Oceanogr., 26, 753-774, doi:10.1175/1520-0485(1996)026<0753:TDOTAC $>2.0 . C O ; 2$.

,,-- B. Sinha, and J.-O. Wolff, 1997: Parameterization of mesoscale eddy fluxes in zonal ocean flows. J. Mar. Res., 55, 1127-1162, doi:10.1357/0022240973224076.

— S. S. Danilov, and D. Olbers, 2008: Eddies in numerical models of the Southern Ocean. Ocean Modeling in an Eddying Regime, Geophys. Monogr., Vol. 177, Amer. Geophys. Union, 177-198, doi:10.1029/177GM13.

_ - B. Sinha, V. B. Zalesny, R. Marsh, and A. T. Blaker, 2013: Influence of bottom topography on integral constraints in zonal flows with parameterized potential vorticity fluxes. J. Phys. Oceanogr., 43, 311-323, doi:10.1175/JPO-D-12-0126.1.

_- S. Danilov, B. Sinha, and J. Schroeter, 2014: Integral constraints for momentum and energy in zonal flows with parameterized potential vorticity fluxes: Governing parameters. J. Phys. Oceanogr., 44, 922-943, doi:10.1175/JPO-D-13-0173.1.

Kamenkovich, V. M., M. N. Koshlyakov, and A. S. Monin, 1986: Synoptic Eddies in the Ocean. D. Reidel Publishing Company, $433 \mathrm{pp}$.

Killworth, P. D., 1992: An equivalent barotropic mode in the Fine Resolution Antarctic Model. J. Phys. Oceanogr., 22, 1379-1387, doi:10.1175/1520-0485(1992)022<1379:AEBMIT>2.0.CO;2.

_ 1997: On the parameterization of eddy transfer. Part I. Theory. J. Mar. Res., 55, 1171-1197, doi:10.1357/0022240973224102.

Krupitsky, A., V. M. Kamenkovich, N. Naik, and M. A. Cane, 1996: A linear equivalent barotropic model of the Antarctic Circumpolar Current with realistic coastlines and bottom topography. J. Phys. Oceanogr., 26, 1803-1824, doi:10.1175/ 1520-0485(1996)026<1803:ALEBMO>2.0.CO;2.

Marshall, D. P., and A. J. Adcroft, 2010: Parameterization of ocean eddies: Potential vorticity mixing, energetics and Arnold's first stability theorem. Ocean Modell., 32, 188-204, doi:10.1016/ j.ocemod.2010.02.001.

_ J. R. Maddison, and P. S. Berloff, 2012: A framework for parameterizing eddy potential vorticity fluxes. J. Phys. Oceanogr., 42, 539-557, doi:10.1175/JPO-D-11-048.1.

Marshall, J. C., 1981: On the parameterization of geostrophic eddies in the ocean. J. Phys. Oceanogr., 11, 257-271, doi:10.1175/1520-0485(1981)011<0257:OTPOGE > 2.0.CO;2.

Maximenko, N. A., B. Bang, and H. Sasaki, 2005: Observational evidence of alternating zonal jets in the world ocean. Geophys. Res. Lett., 32, L12607, doi:10.1029/2005GL022728.
McWilliams, J. C., and J. S. Chow, 1981: Equilibrium geostrophic turbulence. I: A reference solution in a beta plane channel. J. Phys. Oceanogr., 11, 921-949, doi:10.1175/ 1520-0485(1981)011<0921:EGTIAR > 2.0.CO;2.

—, W. R. Holland, and J. S. Chow, 1978: A description of numerical Antarctic Circumpolar Currents. Dyn. Atmos. Oceans, 2, 213-291, doi:10.1016/0377-0265(78)90018-0.

Olbers, D., 2005: On the role of eddy mixing in the transport of zonal ocean currents. Marine Turbulence: Theories, Observations, and Models, H. Baumert, J. Simpson, and J. Sündermann, Eds., Cambridge University Press, 511-546.

Pedlosky, J., 1964: The stability of currents in the atmosphere and the ocean: Part I. J. Atmos. Sci., 21, 201-219, doi:10.1175/ 1520-0469(1964)021<0201:TSOCIT>2.0.CO;2.

Rhines, P., 1975: Waves and turbulence on a $\beta$-plane. J. Fluid Mech., 69, 417-443, doi:10.1017/S0022112075001504.

Ringler, T., and P. Gent, 2011: An eddy closure for potential vorticity. Ocean Modell., 39, 125-134, doi:10.1016/ j.ocemod.2011.02.003.

Stevens, D. P., and V. O. Ivchenko, 1997: The zonal momentum balance in an eddy-resolving general-circulation model of the Southern Ocean. Quart. J. Roy. Meteor. Soc., 123, 929-951, doi:10.1002/qj.49712354008.

Treguier, A. M., I. M. Held, and V. D. Larichev, 1997: Parameterization of quasigeostrophic eddies in primitive equation ocean models. J. Phys. Oceanogr., 27, 567-580, doi:10.1175/ 1520-0485(1997)027<0567:POQEIP > 2.0.CO;2.

Wardle, R., and J. Marshall, 2000: Representation of eddies in primitive equation models by a PV flux. J. Phys. Oceanogr., 30, 2481-2503, doi:10.1175/1520-0485(2000)030<2481: ROEIPE $>2.0 . \mathrm{CO} ; 2$.

Welander, P., 1973: Lateral friction in the ocean as an effect of potential vorticity mixing. Geophys. Fluid Dyn., 5, 173-189, doi:10.1080/03091927308236114.

Wolff, J.-O., E. Maier-Reimer, and D. J. Olbers, 1991: Wind-driven flow over topography in a zonal $\beta$-plane channel: A quasigeostrophic model of the Antarctic Circumpolar Current. J. Phys. Oceanogr., 21, 236-264, doi:10.1175/1520-0485(1991)021<0236: WDFOTI $>2.0 . \mathrm{CO} ; 2$.

Wood, R. B., and M. E. McIntyre, 2010: A general theorem on angular-momentum changes due to potential vorticity mixing and on potential-energy changes due to buoyancy mixing. J. Atmos. Sci., 67, 1261-1274, doi:10.1175/ 2009JAS3293.1. 